\title{
Low Levels of Cephalosporine Resistance and high Azithromycin Resistance in Neisseria gonorrhoeae in Germany
}

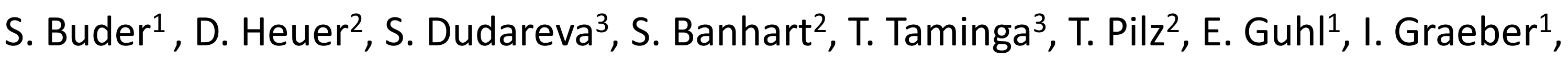 \\ P.K. Kohl' ${ }^{1}$, V. Bremer ${ }^{3}$, K. Jansen ${ }^{3}$

\begin{abstract}
1 German Reference Laboratory for Gonococci, Department of Dermatology and Venerology, Vivantes Hospital Berlin, Germany 2 Unit Sexually Transmitted Bacterial Infections, Robert Koch Institute, Berlin, Germany

3 Department for Infectious Disease Epidemiology, Robert Koch Institute, Berlin, Germany
\end{abstract}

\section{Background:}

The widespread antimicrobial resistance of Neisseria gonorrhoeae (NG) is a serious problem for the treatment and control of gonorrhoea. Many of the previously effective therapeutic agents are no longer viable. Because NG infections are not reportable in Germany, only limited data on disease epidemiology and antimicrobial susceptibility patterns are available. The Gonococcal Resistance Network (GORENET) is a surveillance protocol to monitor trends in the antimicrobial susceptibility of NG in Germany and link this to epidemiological data and NG multiantigen sequence typing data to guide treatment algorithms and target future prevention strategies.

\section{Methods:}

Between April 2014 and December 2017, data on patient-related information were collected from laboratories nationwide and susceptibility testing was performed on $1576 \mathrm{~N}$. gonorrhoeae isolates forwarded from the network laboratories to the Reference Laboratory for Gonococci. Susceptibility results for cefixime, ceftriaxone, azithromycin, ciprofloxacin and penicillin were defined according to EUCAST 4.0 standards. Percentages, medians and interquartile ranges (IQR) were calculated. NG multiantigen sequence typing (NG-MAST) was performed for a third of these isolates.

\section{Results:}

In total 23 laboratories submitted data and 1576 isolates were tested. Altogether, 90\% of isolates were from men. The median age was 32 (IQR 25-54) years for men and 25 (IQR 22-40) years for women (pvalue $<0.001)$. The most frequently tested materials among men were urethral (96.1\%) and rectal swabs (1.7\%), and endocervical and vaginal swabs $(84.3 \%)$ among women.

+ None of the isolates was resistant against ceftriaxone (MIC $>0.125 \mathrm{mg} / \mathrm{L}$ ).

+ Cefixime resistance (MIC>0.125 mg/L) ranged between $2.1 \%$ (2016) and $1.0 \%$ (2017).

One cefixime resistant isolate $(\mathrm{MIC}=0.25 \mathrm{mg} / \mathrm{L}$ ) displayed further resistance for azithromycin and ciprofloxacin, intermediate susceptibility to penicillin and showed reduced susceptibility to ceftriaxone at breakpoint MIC $0.125 \mathrm{mg} / \mathrm{L}$.

+ Resistance against azithromycin was increased in 2014/15 (9.8\% and $11.9 \%$ ) but decreased to $4.3 \%$ in 2016 and $3.9 \%$ in 2017. In 2015 one isolate displayed high-level resistance (> 256mg/L) to azithromycin.

+ Rates of resistance to ciprofloxacin $(72 \%$ in $2014,56 \%$ in 2015 , $53.4 \%$ in 2016 and 63.7 in 2017) and plasmid-mediated high-level penicillin resistance (PPNG) (25.1\% in 2014 to $16.1 \%$ in 2017) are high across Germany.

+ Selected isolates underwent Multiantigen Sequence Typing (NG-MAST) analysis and showed a predominance of ST1407 in $2014 / 2015$ with decreasing number during observation period and a wide range of other STs, including various new sequence types. (see: Poster ID:370, Molecular Typing of Neisseria gonorrhoeae Strains in Germany (2014 -2016) by Neisseria gonorrhoeae Multiantigen Sequence Typing NGMAST)

\section{Conclusion:}

Resistance to ceftriaxone was not detected in Germany and the percentage of isolates with resistance to cefixime was low. Azithromycin resistance showed a discontinuous trend with partly high levels during the observation period. Furthermore we detected the first case of a high-level azithromycin resistant NG in Germany in 2015. In 2016/17 azithromycin resistance decreased. This development needs further observation.

The rates of ciprofloxacin resistance and penicillin resistance were high across Germany.

Multidrug-resistance was not detected regularly in GORENET. Nevertheless, the combination of resistances is particularly alarming and should be further monitored.

Continued surveillance of antimicrobial drug susceptibilities for NG remains mandatory to ensure efficient disease management.

Disclosure of Interest Statement: GORENET is funded by German Federal ministry of health. No conflicts of interest. 